\title{
Analysis and Ideas for Improved Routing in MANET
}

\author{
https://doi.org/10.3991/ijim.v13i04.9928 \\ J. P. Josh Kumar ( $\left.{ }^{凶}\right)$ \\ Sathyabama University, Chennai, India \\ Agni College of Technology, Chennai, India \\ josh.kumar.vlsi@gmail.com \\ A.Kathirvel \\ Misrimal Navajee Munoth Jain Engineering College, Chennai, India
}

\begin{abstract}
Mobile Ad hoc NETwork is a collection of mobile nodes connected with each other without any centralized infrastructure support. In MANET, every node acts as a router, thereby forwards the packet to other nodes through the best route possible. The cooperation of all other nodes is very essential for a node to transfer data to its desired destination. Throughput is a major factor that determines the health of any network including MANET and there can be many reasons that can disrupt the throughput. In this paper we deal with two prime reasons that may reduce the throughput in a MANET. The first reason is that, if a node behaves selfishly and utilizes the path only for forwarding its own packet, then the packets from other nodes will be dropped and the throughput will gradually get reduced. The second reason for reduction in throughput is the phenomenon called fading, which causes signal attenuation. This phenomenon creates path loss temporarily, during which the packet loss increases and the throughput takes a major hit. Removing such nodes from the network on the basis of doubt about its packet forwarding nature and ability will eventually weaken the network strength. Neglecting the original best route will also reduce the quality of the network. Both the cases of selfishness and fading can be normalized by switching over to an alternate path and coming back to the same route after a short period of time. Thereby the quality as well as the quantity of nodes involved in the network can be maintained. In this paper we have also introduced an innovation called helper nodes which will also help in the formation of alternate path and will very well neutralize the ill effects caused due to selfishness and fading.
\end{abstract}

Keywords-MANET, selfish nodes, fading, throughput, alternate route, helper nodes.

\section{$1 \quad$ Introduction}

In recent years, Mobile ad hoc networks have witnessed various areas of research to improve its performance and one such is to improve the quality of routing. The routing protocol for MANET can be classified as reactive and proactive. In reactive routing protocol the route information is generated only when there is a necessity to 
transmit data from a source to destination. Whereas, in proactive routing, the network's routing information is constantly updated in all its nodes irrespective of data transmission [1]. We have considered the Ad-hoc on-demand distance vector routing (AODV) the most popular reactive routing protocol for our analysis [2].

In terms of speed, proactive routing is better as we can form the route in lesser time as the route information is constantly updated and readily available. But, the drawback here is that as the routes are constantly updated, this will create lots of network overhead. Whereas in reactive routing protocol, the routing will take comparatively a little longer time as the paths are traced only when there is a need for transmission of data but here the network overhead is very minimum [3]. There is always a tradeoff between speed and overhead while selecting the type of routing.

MANET is a collection of battery powered mobile nodes with less bandwidth, so we need to keep the transfer of frequent "hello packets" between the nodes to a minimum. By doing this we can reduce the usage of battery as well as bandwidth, which can be achieved in reactive routing protocol. Based on this consideration, we have selected the reactive routing approach.

The wireless medium is always risk prone as there might be various security issues. Apart from the medium even the participating nodes can disrupt the wireless network by indulging in harmful activities like Black Hole attack, Byzantine attack, Snooping attack, Man-in-the-middle attack and the list is endless [4]. Considering the above statement the nodes participating in the ad hoc network can be classified as helpful and harmful, based on their behavior. Helpful nodes are the pillars of the network and they help neighboring nodes by forwarding their packets. Harmful nodes are not cooperative and in times they try to disrupt the entire network.

Among the harmful nodes there are a certain category of nodes which will not cause any intentional harm to the network but will have a temporarily self interested behavior to protect its own resources like battery and bandwidth. They may use the network to forward its own data packets at times. The main reason for this selfish behavior is its reducing battery power [5]. In this era of power harvesting devices, these nodes should be given some time so that it can harvest power and come back to its original selfless behavior. The fading is also a reason for the loss of signal power or fluctuations in the received power. In the case of multipath fading the signal will again regain its original strength or even higher level of received power can be expected.

Therefore it is evident that we need to wait for a certain amount of time before classifying any node as a selfish node or switching to another route altogether. Meanwhile the network operations should be continued without any further loss of throughput and increase in delay. Therefore, in this paper, we deal with two prime reasons that may reduce the throughput in a MANET and we have also tried to establish the conditions for selecting the alternate path and switching back to the initial optimum path. 


\section{Related Work}

In Mobile ad hoc networks, many research works have focused on the selfish node problem, as the network connectivity will be greatly affected by the selfish nodes [6]. Most of the works are built on a mechanism called the watchdog. It consists of two parts:

- Watchdog

- Pathrater

Watchdog enabled nodes listen to other nodes transmission a if it transmits within a given time it increases the trust count else the count will be decreased. If the count of any node goes below a predefined threshold value, the particular node will not be used in other routing process, at least for a particular time interval [7].

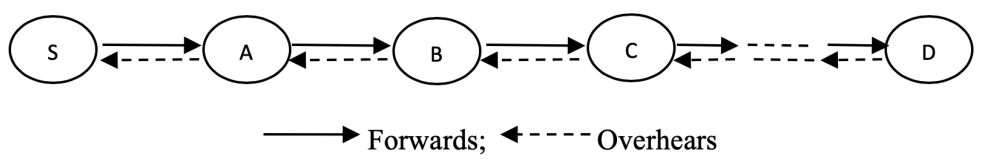

Fig. 1. Watchdog working mechanism

Here node-A maintains a buffer of recently sent packets to node-B and node-A compares each overheard packet from node-B's transmission with the packets in the buffer and removes the matching packet from the buffer, If a packet remains in the buffer too long, it increases the failure tally of node-B, if the tally exceeds a threshold value, then node- $\mathrm{B}$ will be marked selfish and the source will be notified, likewise node-B will act as a watchdog for node-C and so on till the destination [7].

The process of rating each node is done by a mechanism called pathrater, which assigns 0.5 to neutral nodes, 0.01 to active nodes and -0.05 to nodes where link break occurs. In this watchdog, mechanism chances are there for a wrong rating on account of collisions occurring at the monitoring or receiver node and also due to limited transmission power of nodes. This false alarm can be avoided when the destination sends an acknowledgement (A) to the source, on receiving the data packet $(\mathrm{P})$ [8].

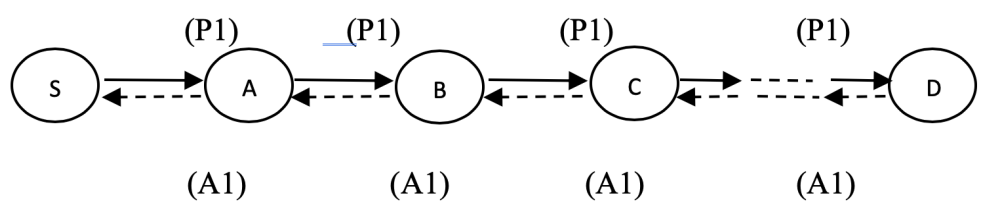

Fig. 2. Acknowledgement technique

This acknowledgement technique increases the overhead, so a modified version called TWOACK (Two Acknowledgement) is introduced. In this each node is required to send back an acknowledgement packet to the node that is two hops away from it, thereby controlling the data overhead [9]. 
Based on watchdog and the acknowledgement technique an umpiring system is proposed, where all nodes have dual roles packet forwarding and umpiring. During RREQ and RREP, each node monitors the performance of its immediate neighbor. If there is any issue in the forwarding of packets by any node that particular node will be booked as a selfish node [10]. This umpiring model was further improved by adding two more neighboring nodes as umpires and the selfish nodes can be very well identified and detained [11].

Fading is the other issue that needs to be analyzed for proper decision making by any MANET routing protocol. The nodes which are part of a link will continuously monitor the other nodes in the link and decides whether to continue sending packets through that node or not. Nodes also decide which node can be included in the link [12]. Fading causes fluctuations in the signal-to-noise ratio, these fluctuations may cause a packet drop in a good link and may also cause a packet to be received though the link is bad. This will cause unnecessary path drop and new path search [13]. Finegrain variations in signal strength should also be taken into account so that the fading can be predicted and balanced through stochastic modeling [14].

In order to extend the network lifetime and balance the battery power of individual nodes, care must be taken to reduce the transmission distance of the nodes. Even when the node is capable of transmitting to longer distance, it should be made to use the nearby nodes to transmit as transmitting to longer distance requires more transmission power. In sensor nodes the same concept is applied by creating clusters and cluster heads for managing power consumption [15]. The hop count increases with increasing number of nodes in the transmission. So in Mobile Ad Hoc Networks with more number of nodes we can try to have shorter distance hops thereby the power consumption can be reduced [16]. One more reason why we need to switch over to the alternate path is that the TTL (Time to live) is limited for the packets during transmission. In that case if we are not switching over to the alternate path, those packets will end up lost or dropped in a no forwarding loop [17].

\section{Effects of Fading in MANET}

Fading is a natural phenomenon where the transmitted signal strength is gradually reduced before it reaches the receiver. There are various reasons for fading, many of which cannot be avoided but can only be managed. The fading effect will cause a dip in signal strength and sometimes it increases the signal strength. The frequency range of Mobile ad hoc network is $30 \mathrm{MHz}$ to $5 \mathrm{GHz}$ this means we can expect a range of .06 meters ( 6 Centimetres) to 10 meters. Reduction of signal strength due to fading will also adversely affect the range of transmission. Thus, once the signal strength goes below a certain threshold value we can switch over to the available alternate path, thereby the data transmission can continue unhindered.

Also the effects of fading will diminish the received power to such a small amount that it appears as if there is no packet transmitted by the neighbouring node. Thus we need to switch over to an alternate path and wait for a fixed amount of time before calling any node, selfish. 


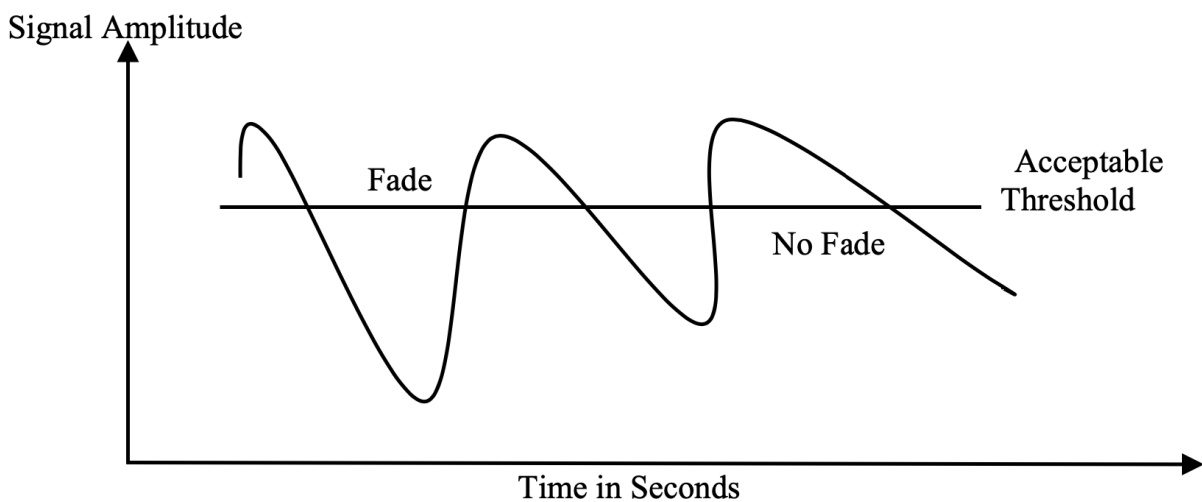

Fig. 3. A model graph showing Signal Fading.

As the basic idea behind MANET is the mobility of its nodes, those nodes can go to any area where there can be many reasons for fading. In such situations we can expect even up to hundred percent fading, thereby a total loss in throughput. Even though we know that the particular route which was followed from source to destination can be revived, once the nodes move back to its original location or at least nearby to the previous location, we have to prevent the temporary loss of throughput. Alternate path is the best available strategy wherein we can move to the second best path and try the best path after a given time interval. Thus the throughput can be maintained by the alternate path and we can also avoid the false positives (due to fading) in our efforts to identify the selfish nodes.

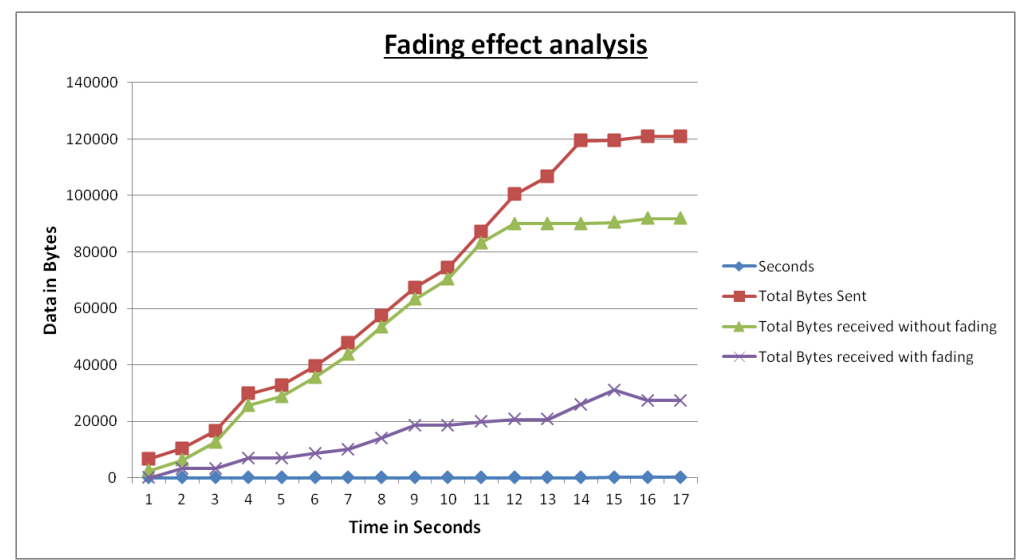

Fig. 4. Rayleigh Fading effect analysis.

Fig. 4 shows an analysis of the effects of fading using the software simulation tool Qualnet. The simulation is done using 15 mobile nodes with random waypoint mobility model and AODV protocol. The channel path loss model is Two Ray, the channel frequency is $2.4 \mathrm{GHz}$, the maximum velocity of nodes is $10 \mathrm{Meters} / \mathrm{Sec}$ and we have 
used Rayleigh fading model. In this graph we can clearly observe how the Rayleigh fading effect impacts the total bytes received by the destination. As the number of bytes becomes low the packet loss is becoming high and the throughput takes a major hit. This will make the particular source node look like a selfish node. Since all the packet loss happening is due to fading, we can go for an alternate route without terming the source as a selfish node. If required the original route can be used after some time interval, in this way we can improve the networks sustainability.

This experiment is repeated in a Cartesian coordinate system terrain of size 1500 * 600 Meters, with two ray path loss model and 100 Mobile Nodes with zero fading and for Rayleigh, Ricean and fast Rayleigh fading. This time we have measured the packets sent and received. Rayleigh fading is a model that can be applied and observed in metropolis where there are many buildings and line of sight propagation is almost not possible. Ricean fading on the other hand is a phenomenon happening in outdoor areas where line of sight propagation plays a more dominant role. Even though Rayleigh and Ricean fading varies in nature, when we did the simulation of MANET using Qualnet we found the packets received for both types of fading to be around only $60 \%$ of the total sent packets. Here we made two observations first is the drastic packet loss and the second is the similarity between the Rayleigh and Ricean fading. The main reason for the similarity between the throughput in case of Rayleigh and Ricean fading may be the highly mobile nature of MANET nodes. This mobility of the nodes in MANET may have neutralized the inherent difference in the nature of Rayleigh and Ricean fading.

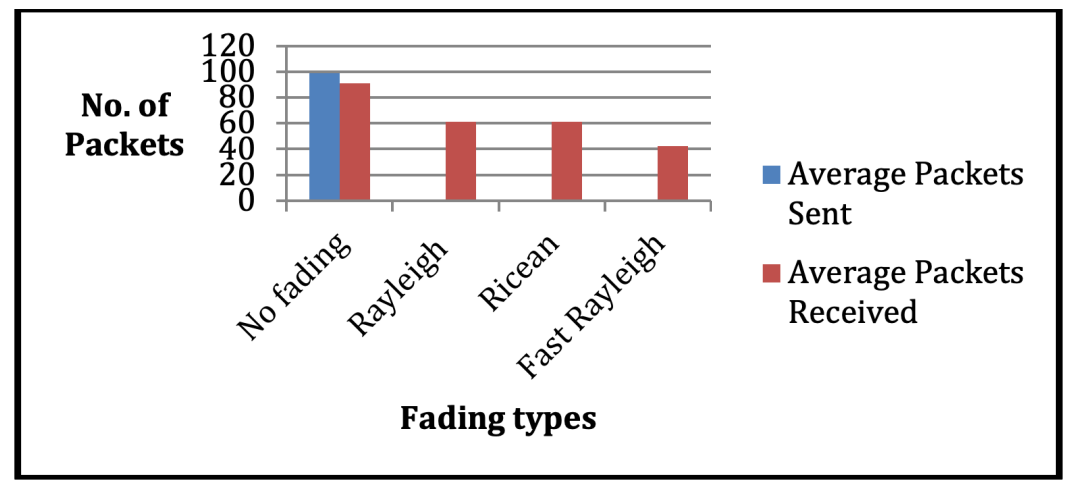

Fig. 5. Fading comparison

We have also observed that the fast Rayleigh fading affects the throughput to a greater extent having only $40 \%$ packet delivery ratio. Fast fading occurs when the coherence time of the channel is small relative to the delay requirement of the application. It originates due to effects of constructive and destructive interference patterns which are caused due to multipath. All these observations as shown in fig. 5 should play an important role in any analysis of MANET routing protocols and should be studied through real time experimentation, to establish more clarity and insights on the effects of fading. This fading is a common issue for all protocols in MANET so 
any solution other than the alternate path solution that results from the efforts of the research community can be treated as a common solution and needs to be implemented in MANET protocols.

\section{Dealing with Selfish Nodes in MANET}

Selfish nodes can be dealt with in many ways. The watchdog, acknowledgement and umpiring techniques help us in finding and eliminating the selfish nodes present. But just by eliminating the selfish nodes without going into the reason for the selfish behaviour will eventually reduce the number of active nodes in the network which will lead to total network failure. Research shows that the main reason for a node to behave selfishly is falling battery level [18]. But with the advent of energy scavenging techniques in Mobile ad hoc network, any node can comeback with a more active selfless participation by helping other nodes [19]. In these scenarios if a node fails to forward a packet to its next node that lies along the route, based on the information received in the Route Request (RREQ) and Route Reply (RREP) phase, the particular node can be given a second chance. When two packets are dropped continuously by any node, i.e. if a node drops packets more than once continuously then the battery power has be monitored and if it is found to be below a prescribed threshold say for example 15 percent of the total power available, then we can switch over to the alternate path.

\section{$5 \quad$ Alternate Path Routing in MANET}

The alternate path has to be deployed when one or more of the below said three conditions exist on the node and the network, one is the battery power, next is the fading effect and the last is the real selfish behaviour. The Route Request (RREQ) packets for searching a new route are generated only when the existing route fails completely due to fading [20]. Thus causing precious information loss in the form of packet loss or drop before the Route Reply (RREP) arrives for creating a new route. In our alternate path approach we continuously monitor the received signal and if the signal falls below a particular threshold value as shown in figure-1, then the data traffic will be routed through alternate path.

The switchover to alternate path can also happen based on the factors like bit error rate (BER), Signal-to-noise ratio (SNR) which varies due to fading. Fading induce variation in the above said factors which can cause two issues one is the discarding of a good route and the other is accepting a bad route. These issues can be sorted out or normalized by temporarily switching over to an alternate path and again coming back to the initial path, when the BER and SNR comes back to acceptable levels. This measure will increase the overall immunity and the network health.

In all the existing MANET routing protocols used in various simulation tools, these factors along with a Morkov prediction mechanism should be incorporated, so that the reliability of the simulation results can be greatly improved. The Morkov model can predict the moment to switch over to the alternate path [21]. In searching and estab- 
lishing the alternate path, node disjoint paths make more sense as we can easily switch over to the alternate path without worrying about the participating nodes.

Markov process is based on the property called memorylessness, which means the future state of a process is based only on the present state and not on the past states. The Markov prediction process can be applied to the fading throughput and also to the throughput without fading. Based on the Markov analysis we can determine whether to switch to the alternate path or not. Similarly the alternate path selected can be of different types namely a disjoint path, availability-based disjoint path and maximally disjoint path. A disjoint path can be further divided into node disjoint path and link disjoint path [22]. In a node disjoint path the path will not share any common node. Similarly in link disjoint path, the path will not have any common links. We can also have a combined model of node and link disjoint paths which is simply called the disjoint path as shown in the fig. 6 and found to be the best option we can have in the alternate path. Here based on the diagram node 25 is the source and the node 6 is the destination and we can see that there are two paths shown in blue and red lines. Both the path doesn't have any common link or common nodes; this is what we call as the disjoint path.

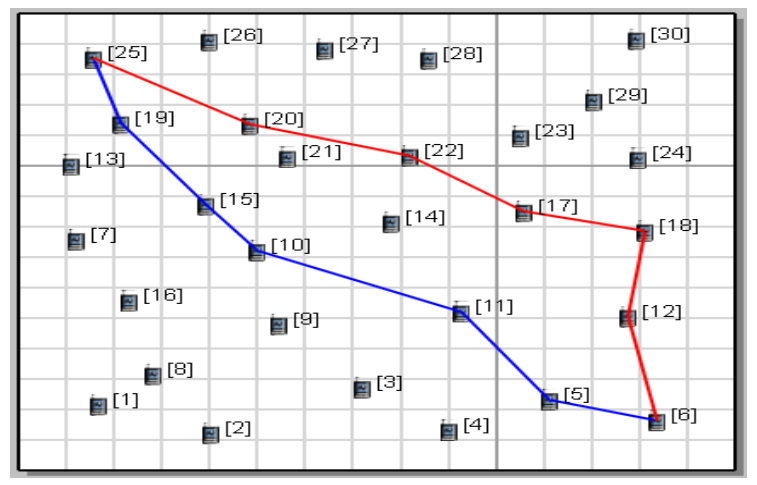

Fig. 6. Disjoint Paths

The availability-based disjoint path is one in which the paths are link and node disjoint but it is limited to the availability constraint. This means some nodes and links may not be disjoint, as such disjoint nodes to cover the entire length from source to destination may not be available. The last is the maximally disjoint path where paths share maximum disjoint nodes and links, but will also have a minimal common nodes and links. All these efforts taken in the establishment of alternate path will definitely improve the connectivity, throughput and stability of the entire network.

\section{An Innovation Called Helper Nodes}

We consider a civilian scenario, where there are a number of people using MANET in a university premises. The people who are in areas or buildings along the perimeter of the university campus are less likely to get affected due to the constant communica- 
tion \& the corresponding battery loss. In the same vein, we must mention that the battery power will be rapidly reducing for those who are in the centre of the campus. The scenario is designed as shown in fig. 7 showing the nodes which are involved in the formation of a mobile ad hoc network and also the helper nodes which are shown in red.

Here in MANET, the main difference it has with the general wireless network is that, there is no centralized fixed infrastructure. But what we can do is, we can setup temporary dummy nodes which will not have any packets or data to be transmitted on its own, but will happily help other nodes in transmitting their packets. These dummy nodes can be called as helper nodes, which will route the packets of the network from source to destination.

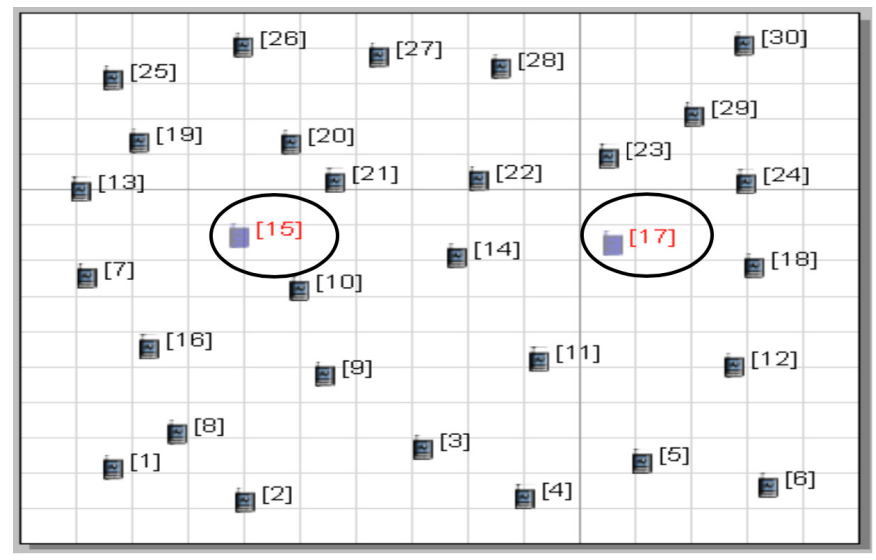

Fig. 7. Helper Nodes

These helper nodes should be placed at the central area of the campus and should be always available for routing. In this scenario as shown in the fig. 7 the helper nodes are placed and numbered as $15 \& 17$, shown in red with a circle around. The helper nodes, is a new concept in MANET, that will help in strengthening the connectivity of the entire network. These helper nodes should be placed at strategic locations to enable uninterrupted continuous service of the MANET. But, one issue that will arise here is that, the helper nodes will more or less be involved in continuous activity, as it lies at the centre of the network. This will cause their batteries to get drained at very faster rates than other nodes. Therefore if the node dies for lack of battery power, the throughput rate will come down and there may even be lack of connectivity temporarily.

If this is allowed to continue, eventually many nodes at the centre of the network will lose its battery power and slowly the network will get collapsed. To avoid all these, we need to switch over to an alternate path whenever the battery of these helper nodes as well as other nodes goes below a set threshold level. And also in this era of self rechargeable batteries, these nodes battery should be connected to solar panel, so that once we leave these nodes undisturbed, power level will slowly limp back to normal safe levels 


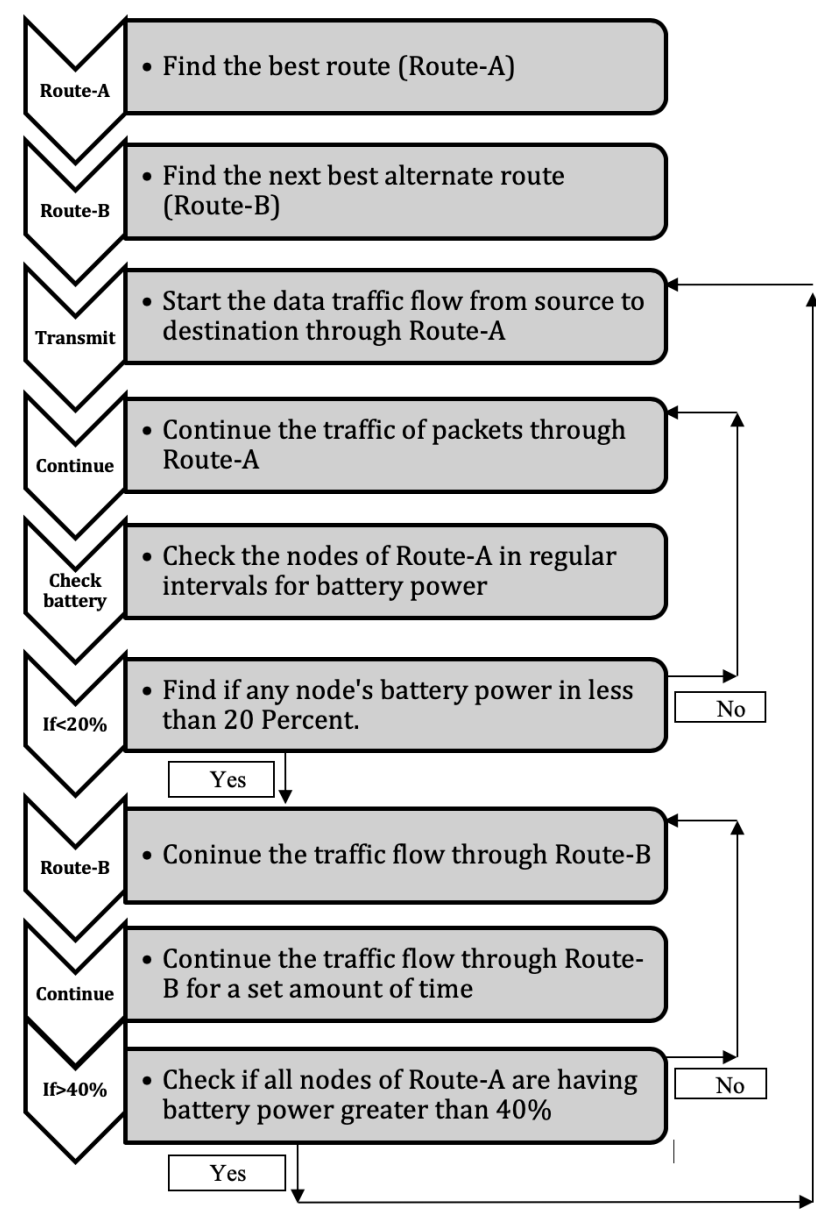

Fig. 8. Flow Chart showing the alternate path logic

Thus the concept of helper nodes and self rechargeable idea combined with the alternate path approach will revolutionize the entire field of Mobile Ad Hoc Networks and boost its deployment in various spheres of everyday life. These helper nodes will also help in the formation of alternate path and will very well neutralize the ill effects caused due to selfishness and fading.

The logic in choosing the alternate path is shown in the fig. 8, where initially, we need to find the best path based on the routing protocol we use and in our example we have used the AODV protocol for all the experimentation purpose. And unlike in AODV here we do an extra step that is we also find the next best route. We may try to go for disjoint path for better results or we can go for availability-based disjoint path. Then the traffic flow from the source to the destination can be done through the best path we have indentified which is also called as Route-A. This flow of traffic from source to destination can be continued through Route-A, as long as all the node is having battery power greater than $20 \%$. For that, a continuously battery power moni- 
toring system should be set up in the routing protocol, and every individual node's battery power should be monitored at regular intervals. When any node's battery power is found to be less than $20 \%$, the routing protocol should automatically switch over to the next best route i.e. Route-B.

While continuing to route traffic through Route-B, after a set amount of time interval, again the nodes of Route-A should be checked for their individual battery power. But, this time the criterion is that every individual node's battery power in Route-A should be greater than $40 \%$. Only if that criteria is satisfied, we can again move back to our original best Route-A, otherwise we can continue the data traffic routing through Route-B and keep checking the battery levels of the nodes of Route-A at regular intervals. Thus the alternate path logic will work well with the existing MANET routing protocols, thereby improving its efficiency in terms of throughput, connectivity, reliability and in many areas of QOS.

\section{Conclusion and Future Scope}

This paper presents a great deal of theoretical insights which will kindle the minds of researchers in the field of MANET and will propel their efforts to strengthen and improve the routing protocols in MANET. The concept of helper nodes will also enrich the adaptability of the MANET routing protocols. Fading and selfishness are the two issues in MANET addressed in this paper and we have given a common solution for both the issues. The solution is, reaching out to the alternate path till a time the fading effect and selfishness like nature of nodes stops. We have simulated the effects of fading and dealt with three types called the Rayleigh, Ricean and fast Rayleigh fading. The selfishness aspect of nodes was dealt in more of a theoretical fashion. The analysis of both fading and selfishness gives a fresh start to a more practical experimentation of these issues, by the research community in the ever evolving field of MANET. Any solution that is found can be commonly implemented for all the reactive routing protocols which are presently at use in MANET.

\section{References}

[1] R Shenbagapriya, and N. Kumar, "A survey on proactive routing protocols in MANETs", In: Proc. Of International Conference on Science Engineering and Management Research, Chennai, India, 2015.https://doi.org/10.1109/ICSEMR.2014.7043630

[2] C.E. Perkins, and E.M. Royer, "Ad-hoc on-demand distance vector routing", In: Proc. of Workshop on Mobile Computing Systems and Applications, New Orleans, LA, USA, 1999.https://doi.org/10.1109/MCSA.1999.749281

[3] Daxesh N. Patel, Sejal B. Patel, Hemangi R. Kothadiya, Pinakin D. Jethwa, and Rutvij H. Jhaveri, "A survey of reactive routing protocols in MANET", In: Proc. Of International Conference on Information Communication and Embedded Systems (ICICES), Chennai, India, 2014.

[4] Rashid Hafeez Khokhar, Md Asri Ngadi, and Satria Mandala, "A Review of Current Routing Attacks in Mobile Ad Hoc Networks", International Journal of Computer Science and Security, Vol.2 No. 3, pp.18-23, 2008. 
[5] J.P.Josh Kumar and A.Kathirvel, "Optimum Power Management in Mobile Ad-Hoc Networks", Journal of Engineering Science and Technology, Vol. 13, No.6, pp.1805-1815, 2018.

[6] Mani Bharathi, Ranjith Sairam, S. Sundar, and C. M. Vidhyapathy, "Securing AODV Protocol from Selfish Node Attack", ARPN Journal of Engineering and Applied Sciences, Vol. 10, No. 12, 2015.

[7] Sergio Marti, T.J. Giuli, Kevin Lai, and Mary Baker, "Mitigating Routing Misbehaviour in Mobile Ad Hoc Networks", In: Proc. of the $6^{\text {th }}$ International Conf. On Mobile Computing and networking, Boston, Massachusetts, USA, pp.255-265, 2000.

[8] Ashish Kumar, Vidya Kadam, Subodh Kumar, and Shital Pawar, "An AcknowledgementBased Approach for the Detection of Routing Misbehaviour in MANETS", International Journal of Advances in Embedded Systems, Vol. 1, No. 1, pp. 04-06, 2011.

[9] Dilip Kumar Thumu, R.Vasavi, and A. Kousar Nikhath, "Detecting Malfunctioning Nodes in Mobile Ad hoc Networks by using EAACK", International Journal of Computer Science and Information Technologies, Vol. 6, No. 5, pp. 4313-4317, 2015.

[10] AAyyaswamy Kathirvel, and Rengaramanujam Srinivasan, "Self Umpiring System for Security in Wireless Mobile Ad Hoc Network", Journal of Wireless Sensor Networks, Vol. 2, pp. 264-266, 2010.https://doi.org/10.4236/wsn.2010.23036

[11] RC. Rajabhushanam, and A. Kathirvel, "System of One to Three Umpire Security System for Wireless Mobile Ad hoc Network" Journal of Computer Science, Vol.7, No.12, pp.1854-1858, 2011.https://doi.org/10.3844/jcssp.2011.1854.1858

[12] jJohn Mullen and Hong Huang, "Impact of Multipath Fading in Wireless Ad Hoc Networks", In: Proc. of Second ACM International Workshop on Performance Evaluation of Wireless Ad Hoc, Sensor, and Ubiquitous Networks, Quebec, Canada, 2005.

[13] John P. Mullen, "A Proposed Method to Estimate Signal to Noise plus Interference in order to improve the performance of Mobile Ad Hoc Network Routing Protocols", In: Proc. of IPSI- Amalfi, Italy, February 17-20, 2005

[14] John P. Mullen, "Efficient Models of Fine-Grain variations in Signal Strength" In: Proc. of OPNETWORK, Washington, DC, 2004.

[15] Debopam Acharya and Hyo-Joo Han, "Advances in Integrated Vehicle Health Monitoring Systems", Interactive Journal of Interactive Mobile Technologies, Vol. 5, No. 3, pp.32-37, July 2011.https://doi.org/10.3991/ijim.v5i3.1671

[16] Saher Manaseer and Ibrahem Alhabash, "Number of Node Estimation in Mobile Ad Hoc Networks", Interactive Journal of Interactive Mobile Technologies, Vol. 11, No. 6, pp.6572, July 2017.https://doi.org/10.3991/ijim.v11i6.6986

[17] Ahmad Nahar Quttoum, "Interconnection Structures, Management and Routing Challenges in Cloud-Service Data Center Networks: A Survey", Interactive Journal of Interactive Mobile Technologies, Vol. 12, No. 1, pp.36-60, Jan 2018,.https://doi.org/10.3991/ijim.v12i1. $\underline{7573}$

[18] S.Shailender Gupta, C. K. Nagpal and Charu Singla, "Impact of Selfish Node Concentration in MANETs", International Journal of Wireless \& Mobile Networks, Vol. 3, No. 2, pp.29-37, April 2011.https://doi.org/10.5121/ijwmn.2011.3203

[19] Kaibin Huang, "Mobile ad hoc networks powered by energy harvesting: Battery-level dynamics and spatial throughput", In: Proc. Of IEEE International Conference on Communications, Budapest, Hungary, 2013. https://doi.org/10.1109/ICC.2013.6655022

[20] Ashish S.Bhalodia, N.B.Gohil and Chirag Bhalodia, "Route Maintenance in AODV Routing Protocol: A Review", International Journal of Advance Engineering and Research Development, Vol. 2, Issue 1, pp.283-287, January 2015. 
[21] Eitan Altman, "Application of Markov Decision Processes in Communication Networks: a Survey, Research Report RR-3984, INRIA. 2000, pp.51.

[22] Farabi Iqbal and Fernando A.Kuipers, "Disjoint Paths in Networks", Wiley Encyclopedia of Electrical and Electronics Engineering, Online Publication, 15 June 2015. https://doi. org/10.1002/047134608X.W8254

\section{Authors}

Mr. J. P. Josh Kumar was born on 13th September 1982 at Palayamkottai, Tamilnadu, India. He received his B.E. degree from Raajas Engineering College, Manonmanium Sundaranar University, Tirunelveli, Tamilnadu, India in 2003 and M.Tech. Degree from Sathyabama Institute of Science and Technology, Sathyabama University, Chennai, India in 2005. He immediately joined as Assistant Professor at Vel Tech Multi Tech Dr.Rangarajan Dr.Sakunthala Engineering College, Chennai and worked there for Six years. Then he joined as Assistant Professor at GKM College of Engineering and Technology, Chennai in 2011 and is currently working there.

He has guided more than 20 U.G and P.G Projects. He has presented 7 papers in various national and international conferences. He has also published a paper in a reputed journal. He has got an award for "Best Teaching Methodology" from Vel Tech Multi Tech Dr.Rangarajan Dr.Sakunthala Engineering College, Chennai. He is pursuing PhD in the area of Mobile Ad Hoc Networks in Sathyabama University, Chennai since December 2013 under the able guidance of Dr. A. Kathirvel, Professor and Head of Computer Science and Engineering at Misrimal Navajee Munoth Jain Engineering College, Chennai.

A. Kathirvel was born in Erode, Tamilnadu, India, received his B.E. degree from V.M.K.V. Engineering College, University of Madras, Chennai, in 1998, M.E. degree from Crescent Engineering College, University of Madras, Chennai, in the year 2002 standing 7th rank in the University. He got University medalist and Best Project Award in his PG Degree studies. His Doctoral Degree from Anna University, Chennai in 2010. He has got teaching, research and administrative experience of more than 20 years in various engineering colleges, autonomous institutions and universities.

He is currently working as Professor and Head of Computer Science and Engineering at Misrimal Navajee Munoth Jain Engineering College, Chennai. He has worked as Lecturer, Senior Lecturer, Assistant Professor, Professor, and Professor \& Head in various institutions. He has published more than 90 papers in national and international conferences and in international journals. He is working as scientific and editorial board member of many journals. He has reviewed dozens of papers in many journals. He has author of three books. He has also published a research monograph from the LAP Lambert Academic Publishing GmbH \& Co., Germany, Europe based on his $\mathrm{Ph} . \mathrm{D}$ thesis titled "Umpiring Security Model and Performance improvement on MANETS", costing 110.35 Euros. His other two books are Introduction to GloMoSim and Prevention of Attacks using Umpiring Security Model for MANETS, LAP Lambert Academic Publishing GmbH \& Co., Germany, Europe.

He is a Life member of the ISTE (India), Senior Member IACSIT (Singapore), Life Member IAENG (Hong Kong), Member ICST (Europe), IAES, Member IEEE and 
ACM. He has given a number of guest lecturers/expert talks and seminars, workshops and symposiums. He has visited Dubai, Abu Dhabi and Oman for presentation of his research papers in various international conferences. His biography was published in 29th edition of Marquis's Who's Who in the World in 2012 issue. He has also guided more than 3 dozen projects (B.E/B.Tech/M.E/M.Tech/MCA) in various engineering colleges. He has given many keynote/invited talks/ plenary lecturers in various national and international conferences and chaired many sessions. His research interests are protocol development for wireless ad hoc networks, security in ad hoc network, data communication and networks, mobile computing, wireless networks and Delay tolerant networks.

Article submitted 2018-11-28. Resubmitted 2019-02-25. Final acceptance 2019-03-12. Final version published as submitted by the authors. 